\title{
The Visual Flow and the Interaction with Computers
}

\section{Luis Miguel Muñoz}

Automatic Control and Computer Engineering Department, Universitat Poletecnica de Catalunya, Spain

*Corresponding author: Muñoz LM, Automatic Control and Computer Engineering Department, Universitat Poletecnica de Catalunya, Spain, Tel: 938967231; E-mail: luis.miguel.munoz@upc.edu

Rec date: March 18, 2015, Acc date: March 20, 2015, Pub date: March 26, 2015

Copyright: ( 2015 Muñoz LM. This is an open-access article distributed under the terms of the Creative Commons Attribution License, which permits unrestricted use, distribution, and reproduction in any medium, provided the original author and source are credited.

\section{Editorial}

Visual Flow can be defined as the amount of visual information that passes in front of the eyes per unit of time. There are studies over visual perception that concludes that the human eye integers the signals during a period of time of 120-125 ms [1]. Thereby, an image that moves remaining visible for a time lower that $125 \mathrm{~ms}$ will appear blurred. Nevertheless, following objects in movement with the eyes reduces the relative speed of the image with respect the retina [2]. An image can be displaced over the retina at 3 degrees per second, without losing acuity. The visualization speed can be augmented by means of conjugate eye movement (saccades or pursuit movements) [3]. The pursuit movements appears when the object moves at less than 100 degrees per second [4], then the eyes follow the movement of the object reducing the relative speed over the retina. During saccades, a rapid displacement of the visual axle between different positions is performed. Due that there is not visual feedback to guide the orientation of the eyes during the movement; the speed can be much higher, up 1000 degrees per second, although penalizing the visual acuity.

The preattentive processing allows humans to process automatically the organization of the visual field. The preattentive processing of the visual information is performed automatically over the whole visual field, detecting basic characteristics of the objects (color, contrast, curvature and size). These characteristics are extracted from the screen into the preattentive system and later are merged with the focalized attention system resulting in coherent objects. The preattentive processing is performed quickly and parallel without the necessity of the focalized attention [5]. Tasks that are considered preattentive can be performed in less than $200 \mathrm{~ms}$ [6], because eyes need $200 \mathrm{~ms}$ to start the movement. A technique that uses the advantage of the preattentive vision is the Rapid Serial Visual Presentation, where words or images are shown in the screen sequentially. These objects can be perceptible if are visible between 200-400 ms [7], although humans have the ability to recognize the presence of a target in less than $100 \mathrm{~ms}$ [6].

Summarizing, humans have a limitation in the information flow that can process, that is to say, the number of pixels per unit of time. There is a maximum tracking speed of an image being displaced and the ocular movement can follow (around 100 degrees per second). Depending on the distance between person and screen, this will be translated to maximum speed of the image. It can be computed that for a person at $400 \mathrm{~mm}$ from the screen, this speed is $700 \mathrm{~mm} / \mathrm{s}$.

On the other hand, in order that the person is able to recognize an image in movement, the eye tracking system requires $100 \mathrm{~ms}$ to start; the image must remain at least $450 \mathrm{~ms}$ to be processed; and $200 \mathrm{~ms}$ to be recognized. Known the dimensions of the screen, it can be computed the maximum theoretic displacement of the image over the screen. For a 17" display, this speed results in $360 \mathrm{~mm} / \mathrm{s}$. Some authors [8] use these relationships in order to calibrate systems with speed dependent automatic zoom.

In any case exists a maximum speed were the objects can be moved over the screen, this depends on every person and the workspace layout with respect the screen monitor. From the point of view of an ergonomic design, the computer interface must be designed in a way that the information flow remains into a controlled parameters in order to reduce stress and workload over the user.

\section{References}

1. Zagier EJS (1997) A human's eye view: Motion blur and frameless rendering. ACM Crossroads 3: 4.

2. Morgan MJ, Benton S (1989) Motion-deblurring in human vision. Nature 340: 385-386.

3. Missal M, Keller EL (2002) Common inhibitory mechanism for saccades and smooth-pursuit eye movements. J Neurophysiol 88: 1880-1892.

4. Krauzlis RJ, Stone LS (1999) Tracking with the mind's eye. Trends Neurosci 22: 544-550.

5. Treisman A (1985) Preattentive Processing in Vision. Computer Vision, Graphics, and Image Processing 31: 156-177.

6. Healey CG, Booth KS, Enns JT (1996) High-Speed Visual Estimation Using Preattentive Processing. ACM Transactions on Human Computer Interaction 3: 107-135.

7. Spence R (2002) Rapid, serial and visual: a presentation technique with potential. Information Visualization 1: 13-19.

8. Cockburn A, Savage J, Wallace A (2005) Tuning and Testing Scrolling Interfaces that Automatically Zoom. Proceedings of ACM CHI 2005 Conference on Human Factors in Computing Systems, USA. 